\title{
Ultrasound-Guided, Robotic Gastrointestinal Stromal Tumor Resection
}

\author{
Gregory Tiesi, MD, Sebastian Eid, MD, Donald McCain, MD, FACS \\ Department of Surgery, Hackensack University Medical Center, Hackensack, NJ, USA (Drs. Tiesi, Eid). \\ Department of Surgical Oncology, Hackensack University Medical Center, Hackensack, NJ, USA (Dr. McCain).
}

\begin{abstract}
Introduction: Despite evolving experience with laparoscopic resections of gastric gastrointestinal stromal tumors (GISTs), there are few data describing robotic resections. Here we describe the robotic removal of a gastric GIST using laparoscopic ultrasonography for tumor localization.
\end{abstract}

Case Description: The patient is a 46-year-old African-American man with a biopsy-proven $3.5 \times 3.5$-cm GIST along the greater curvature of the stomach, and with no evidence of metastatic disease on preoperative computed tomographic staging. Three robotic ports (12-mm umbilical, $8-\mathrm{mm}$ left lower quadrant, and 8-mm subxiphoid) were combined with a $12-\mathrm{mm}$ right lower quadrant assist port and a right midabdomen $8-\mathrm{mm}$ port for the laparoscopic liver retractor. Mass localization was facilitated by intraoperative ultrasonography and dissection performed with bipolar electrocautery via dissecting forceps. Resection was performed using a stapled technique and the specimen removed via the assist port. Total operative time was 104 minutes and estimated blood loss was $25 \mathrm{~mL}$. There was no significant morbidity. Length of stay was 3 days, and the patient returned to work within 1 week. The retrieved specimen was CD117- and DOG1-positive with 2 to 3 mitoses/hpf. At 1-year follow-up, there was no evidence of disease.

Discussion: This case report describes a novel and efficient technique for the robotic removal of a gastric GIST. This resection can be safely performed with the aid of intraoperative ultrasonography instead of gastroscopy or gastrotomy for identification of resection margins.

Key Words: Gastrointestinal stromal tumor, Robotics, Laparoscopic ultrasonography.

Citation Tiesi G, Eid S, McCain D. A novel ultrasound-guided, robotic gastrointestinal stromal tumor resection. CRSLS e2014.00120. DOI: 10.4293/CRSLS.2014.00120.

Copyright (C) 2014 SLS This is an open-access article distributed under the terms of the Creative Commons Attribution-Noncommercial-ShareAlike 3.0 Unported license, which permits unrestricted noncommercial use, distribution, and reproduction in any medium, provided the original author and source are credited.

Address correspondence to: Gregory Tiesi, MD, Department of Surgery, UMDNJ - New Jersey Medical School, 150 Bergen Street, Suite E-401, Newark, NJ 07101

Telephone: (973) 580-7660, Fax (973) 972-6591, E-mail: gregorytiesi@gmail.com

\section{INTRODUCTION}

Although gastrointestinal stromal tumors (GISTs) are the most common gastrointestinal tumor of mesenchymal origin, they are relatively uncommon, encompassing $<1 \%$ of gastrointestinal tumors with an annual incidence of 3300 to 6000 in the United States. ${ }^{1}$ The stomach is the most common site of origin of these tumors, yet GISTs comprise $<3 \%$ of gastric malignancies. $^{2}$ Traditionally, chemotherapy has had particularly poor results and adjuvant Gleevec (Novartis Pharmaceuticals, East Hanover, New Jersey, USA), which has shown improved survival, is not effective as a sole therapy for GISTs. ${ }^{3}$ Therefore, surgical resection is the only therapy with plausible curative intent, but the rarity of these lesions makes the development of novel surgical techniques a considerable challenge.

Despite evolving experience with laparoscopic resections of gastric GISTs, there are very few data describing robotic resections. Here we present a case report of a robotic minimally invasive resection of a gastric GIST using laparoscopic ultrasonography for tumor localization.

\section{MATERIALS AND METHODS}

\section{Patient}

The patient is a 46-year-old African American man with no significant past medical history who presented to an outside 


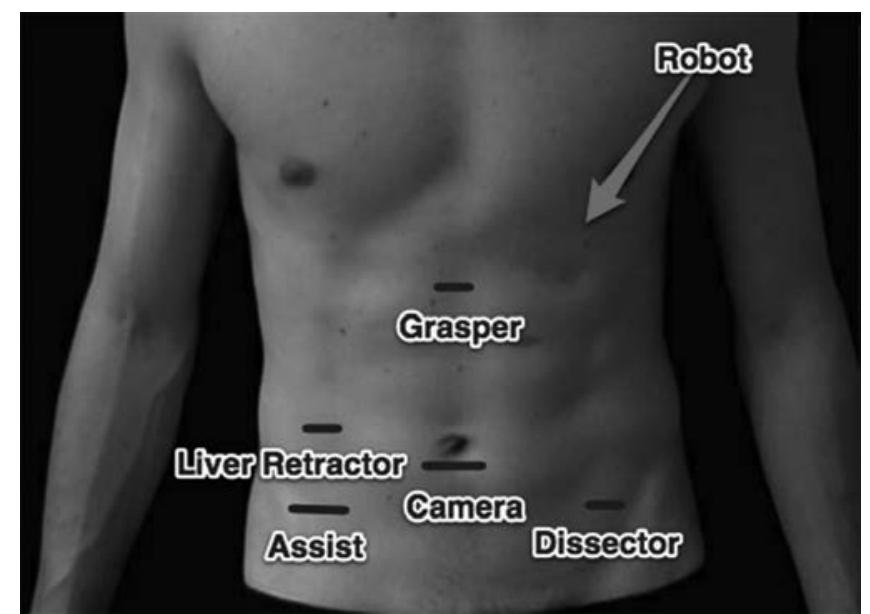

Figure 1. Three robotic ports (grasper: $8 \mathrm{~mm}$, camera: $12 \mathrm{~mm}$, and dissector: $8 \mathrm{~mm}$ ) are combined with one laparoscopic assist port $(12 \mathrm{~mm})$ and one port for the liver retractor $(8 \mathrm{~mm})$.

institution with primary symptoms of melena and syncope. Esophagoduodenoscopy showed a submucosal mass along the proximal to midgreater curvature of the stomach, which was biopsy-proven to be a GIST. Computed tomographic staging confirmed the presence of a $3.5 \times 3.5$-cm mass along the greater curvature without evidence of distant metastasis.

\section{Surgical Technique}

The da Vinci Robotic Surgical System (Intuitive Surgical, Santa Monica, California, USA) was used for this with the patient minimally invasive approach. The procedure was performed under general anesthesia and in the supine position. Both the primary and assistant surgeons were situated to the patient's right side. A $12-\mathrm{mm}$ infraumbilical port was placed using a Veress needle technique, and the abdomen was insufflated to $15 \mathrm{~mm} \mathrm{Hg}$. Laparoscopy was performed to confirm the absence of metastatic disease and to direct both robotic and assist port placement. The robot was docked over the patient's left shoulder. The middle arm of the robot was attached to the laparoscope, and two additional 8-mm robotic ports were inserted under direct visualization: one in the left lower quadrant midclavicular line, which used the robot's left arm, and the other in the subxiphoid position, which used the robot's right arm. A 12-mm nonrobotic port for the first assistant was placed in the right lower quadrant midclavicular line. Just superiorly, another right-sided midclavicular 8-mm port for the liver retractor was inserted (Figure 1).

The laparoscopic liver retractor was inserted to facilitate exposure of the stomach. The dissection was performed using a combination of bipolar electrocautery and blunt

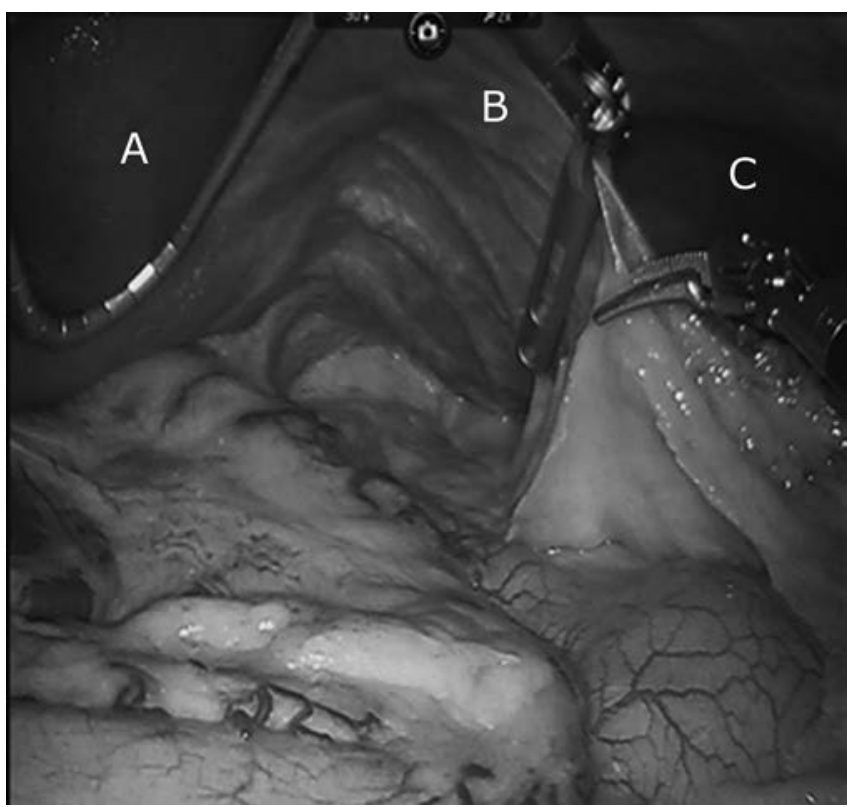

Figure 2. The laparoscopic liver retractor (A) elevates the liver edge as the grasper (B) provides countertraction for the bipolar dissector (C).

dissection via the PK Dissecting Forceps (Intuitive Surgical), with retraction via atraumatic grasping forceps (Figure 2).

The stomach was retracted superiorly using the grasping forceps, and the greater omentum was divided along the greater curvature, using the dissecting forceps. The assistant surgeon provided countertraction with the grasping forceps via the right lower quadrant assist port.

After mobilization of the stomach, the mass was identified and the margins were delineated using a flexible robotic ultrasound probe (Hitachi-Aloka Medical Ltd., Wallingford, Connecticut, USA) inserted through the assist port. The grasping forceps were used to position the lesion in apposition with the ultrasound probe. Using this technique, the lesion was easily identifiable. Conveniently, the ultrasonographic images project directly onto the same screen as the camera images, which greatly improved the efficiency of its use (Figure 3).

An Endo-GIA stapler (Covidien, Mansfield, Massachusetts, USA) was inserted through the assist port and Tri-Staple (Purple, 60-mm cartridge: 3/3.5/4-mm staples) cartridges were used to serially divide the stomach (Figure 4). The ultrasound probe was reinserted as needed through the assist port during staple reloading to verify appropriate margins. A collection bag was inserted through the assist port and the specimen was removed. The specimen was labeled for orientation and ultrasonography was once 


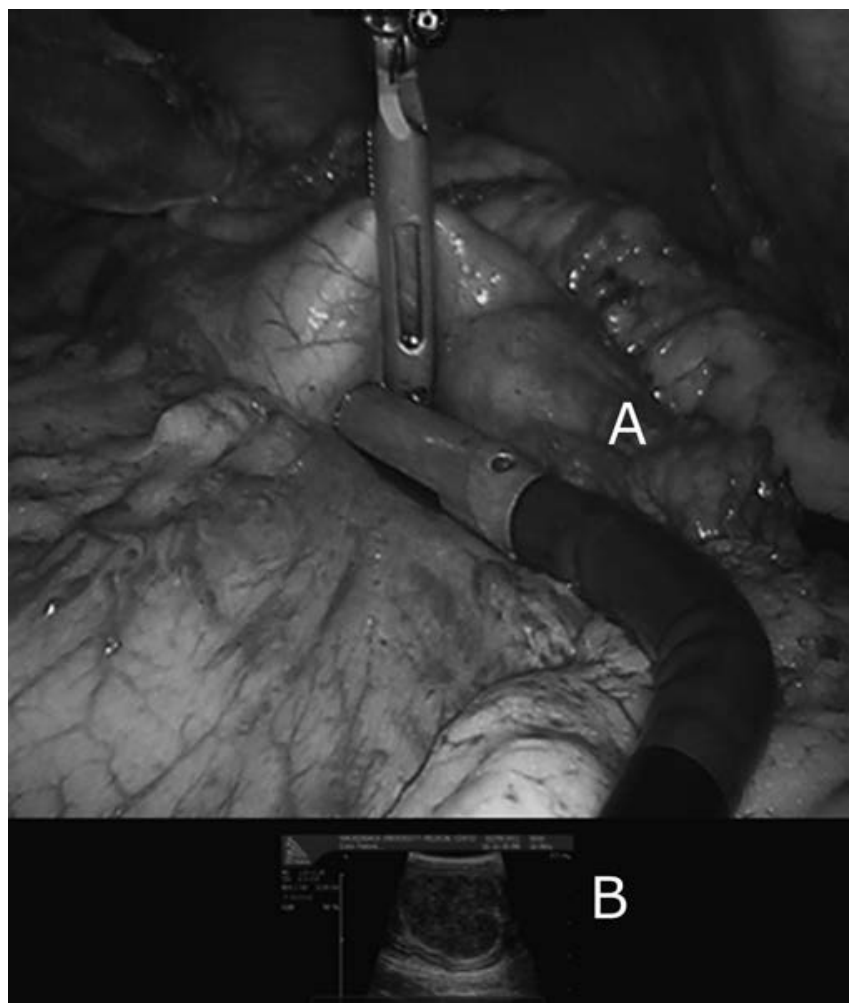

Figure 3. Flexible ultrasound probe (A) images project just below the camera images (B), making the tumor margins readily identifiable.

again used to confirm that the margins were not grossly violated. The abdomen was reinspected to confirm adequate hemostasis. The ports were removed sequentially and closed in a typical manner.

\section{RESULTS}

The total operative time was 104 minutes, with an estimated blood loss of $25 \mathrm{~mL}$. The patient had no significant postoperative morbidity, was able to tolerate a regular diet within 2 days, and was discharged home on postoperative day 3. In addition, the patient was able to return to work within 1 week. Pathologic review of the specimen confirmed a GIST that was CD117- (c-Kit protein) and DOG1positive, with a mitotic rate of 2 to 3 mitoses/hpf. All surgical margins were clear. At 1-year follow-up, there was no evidence of disease.

\section{DISCUSSION}

Laparoscopic resections of gastric GISTs are well-described in the literature. En bloc/R0 resections, avoidance of tumor rupture with minimal manipulation, and maintenance of

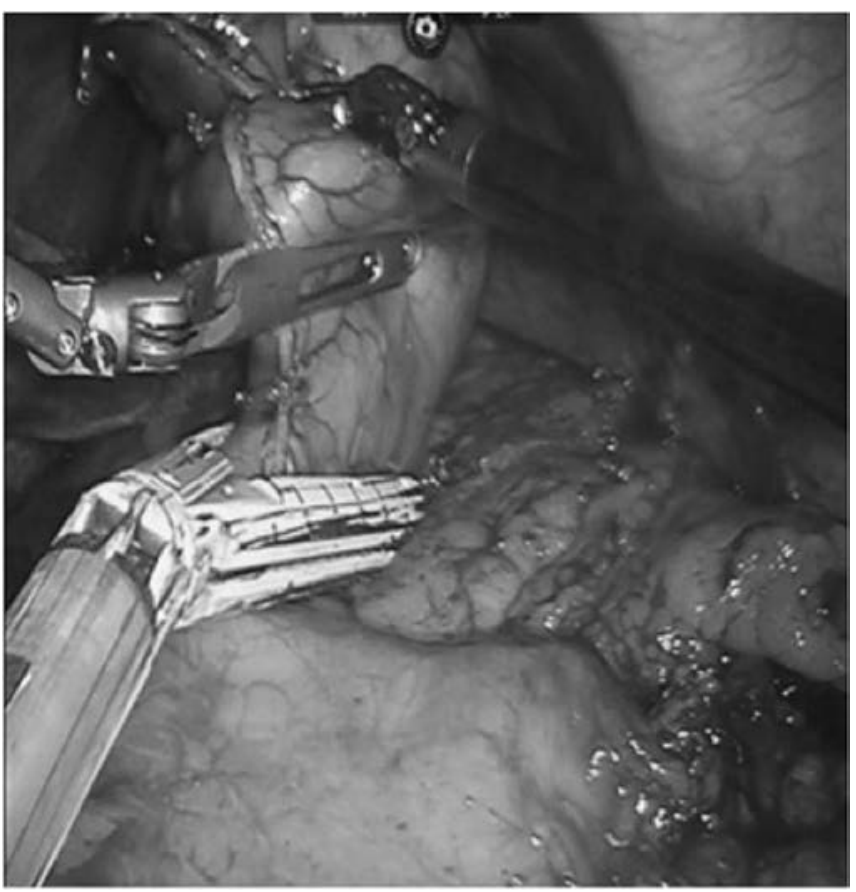

Figure 4. Robotic graspers elevate the lesion as the assistant surgeon inserts a linear stapler through the assist and divides the stomach. Margins are reverified with the ultrasound during stable reloadings.

gastric reserve by minimizing excessive margins are the primary goals. In 2007, the National Comprehensive Care Network recommendations for laparoscopic resections were for tumors $\leq 2 \mathrm{~cm}$ in size with favorable location. ${ }^{4}$ The updated 2010 guidelines, after increased experience with minimally invasive resections, are that tumors $<5 \mathrm{~cm}$ can be removed laparoscopically. Tumors $>5 \mathrm{~cm}$ can be resected laparoscopically if both a favorable location and shape of the tumor exist but may require a hand-assist port. ${ }^{3}$ These recommendations are based in part on several recent series showing that laparoscopic resections can be performed with minimal morbidity and without increased recurrence or incidence of tumor spillage. ${ }^{5-8}$

Despite these advances, there are inherent pitfalls to be considered with a laparoscopic approach, which may be prevented with the use of robotic instruments. The greatest theoretical risk is the aforementioned tumor rupture and seeding with excessive manipulation. Although an uncommon complication, the incidence may increase as we become more aggressive with the lesions we attempt to remove minimally invasively. Specifically, lesions at the GE junction or along the posterior wall pose a significant technical challenge and increase the likelihood of conversion to an open procedure. ${ }^{9}$ Likewise, manipulation of larger lesions, 
even with favorable anatomy, can be hazardous, and the improved dexterity of the robotic instruments can greatly facilitate removal of these lesions.

To date, case reports and series describing robotic resections of gastric GISTs make up a total of 7 patients in the literature. These often involve tumor localization with endoscopy, transgastric resection requiring gastrotomy for visualization, and suture closure of the defect. ${ }^{10-12} \mathrm{Al}-$ though effective, this method may be time-consuming in finding the lesion and closing the defect. It also requires a second invasive procedure and additional personnel and it adds risk, cost, and time.

The concurrent use of intraoperative ultrasonography makes localization of the tumor straightforward and rapid. Surgical margins can be identified and reassessed throughout the resection. The need for endoscopy and gastrotomy for tumor localization is obviated, as well as for intracorporeal suture gastrotomy repair. In addition, the robotic instruments afford the surgeon the ability to freely manipulate the lesion in apposition with the ultrasound probe, therefore optimizing the image and allowing for precise delineation of tumor borders without the need for intraoperative endoscopy. A stapled resection can then be performed without concern for violation of tumor capsule or excessive reduction of gastric volume from overly generous margins.

The combination of these factors permitted a relatively short operating room time (104 minutes), which compared favorably with previously described robotic cases, with operative times approximating 200 minutes. ${ }^{10-12} \mathrm{Al}-$ though variances in tumor anatomy, location, and reconstruction make direct comparison inappropriate, it is certainly compelling to consider.

One notable limitation to this robotic approach is the potential for increased cost. Compared with open surgery, laparoscopic resections for GISTs have been associated with less pain and shorter hospital stays. ${ }^{9}$ Theoretically, in patients in whom a laparoscopic resection could not be safely performed, but a robotic resection could, the increased operative cost may be offset by lesser overall hospital costs or may be justifiable by the decreased morbidity of a minimally invasive approach. For lesions that can be identified without intraoperative endoscopy, have favorable anatomy, and can be resected without excessive manipulation, a traditional laparoscopic approach may be more appropriate from a cost-benefit perspective.

In summary, we have shown that a totally minimally invasive, robotic resection of a gastric GIST can be safely performed. We have also shown that intraoperative ultra- sonography is a novel adjunct that decreases operating room time, obviates the need for gastroscopy/gastrotomy, and facilitates a rapid stapled resection. We feel that this technique is an important technical advancement for minimally invasive resections of gastric GISTs, especially as we stretch the boundaries of which lesions may be considered for a minimally invasive approach.

\section{References:}

1. General Information About Gastrointestinal Stromal Tumors Treatment. http://www.cancer.gov/cancertopics/pdq/treatment/ gist/HealthProfessional/page1. Accessed July 9, 2012.

2. Davis GB, Blanchard DK, Hatch GF, et al. Tumors of the stomach. World J Surg. 2000;24(4):412-420.

3. Demetri GD, von Mehren M, Antonescu CR, et al. NCCN Task Force report: update on the management of patients with gastrointestinal stromal tumors. J Natl Compr Cancer Netw. 2010;8:S1-S41.

4. Demetri GD, Benjamin RS, Blanke CD, et al. NCCN Task Force report: Management of patients with gastrointestinal stromal tumor (GIST) - update of the NCCN clinical practice guidelines. J Natl Compr Cancer Netw. 2007;2:S1-S29.

5. Otani Y, Furukawa T, Yoshida M, et al. Operative indications for relatively small $(2-5 \mathrm{~cm})$ gastrointestinal stromal tumor of the stomach based on analysis of 60 operated cases. Surgery. 2006; 139:484-492.

6. Nakamori M, Iwahashi M, Nakamura M, et al. Laparoscopic resection for gastrointestinal stromal tumors of the stomach. Am J Surg. 2008;196:425-429.

7. Nishimura J, Nakajima K, Omori T, et al. Surgical strategy for gastric gastrointestinal stromal tumors: laparoscopic vs. open re-section. Surg Endosc. 2007;21:875-878.

8. Novitsky YW, Kercher KW, Sing RF, et al. Long-term outcomes of laparoscopic resection of gastric gastrointestinal stromal tumors. Ann Surg. 2006;243:738-745; discussion 745-747.

9. Kong SH, Yang HK. Surgical treatment of gastric gastrointestinal stromal tumor. J Gastric Cancer. 2013;13(1):3-18.

10. Ortiz-Oshiro E, Exposito PB, Sierra JM, Gonzalez JD, Barbosa DS, Fernandez-Represa JA. Laparoscopic and robotic distal gastrectomy for gastrointestinal stromal tumour: case report. Int J Med Robotics Comput Assist Surg. 2010;8(4):491-495.

11. Moriyama H, Ishikawa N, Kawaguchi M, Hirose K, Watanabe G. Robot-assisted laparoscopic resection for gastric gastrointestinal stromal tumor. Surg Laparo Endo Per. 2012;22(3):e155-e156.

12. Buchs NC, Bucher P, Pugin F, Hagen ME, Morel P. Robotassisted oncologic resection for large gastric gastrointestinal stromal tumor: a preliminary case series. J Laparoendosc Adv Surg Tech. 2010;20(5):411-415. 